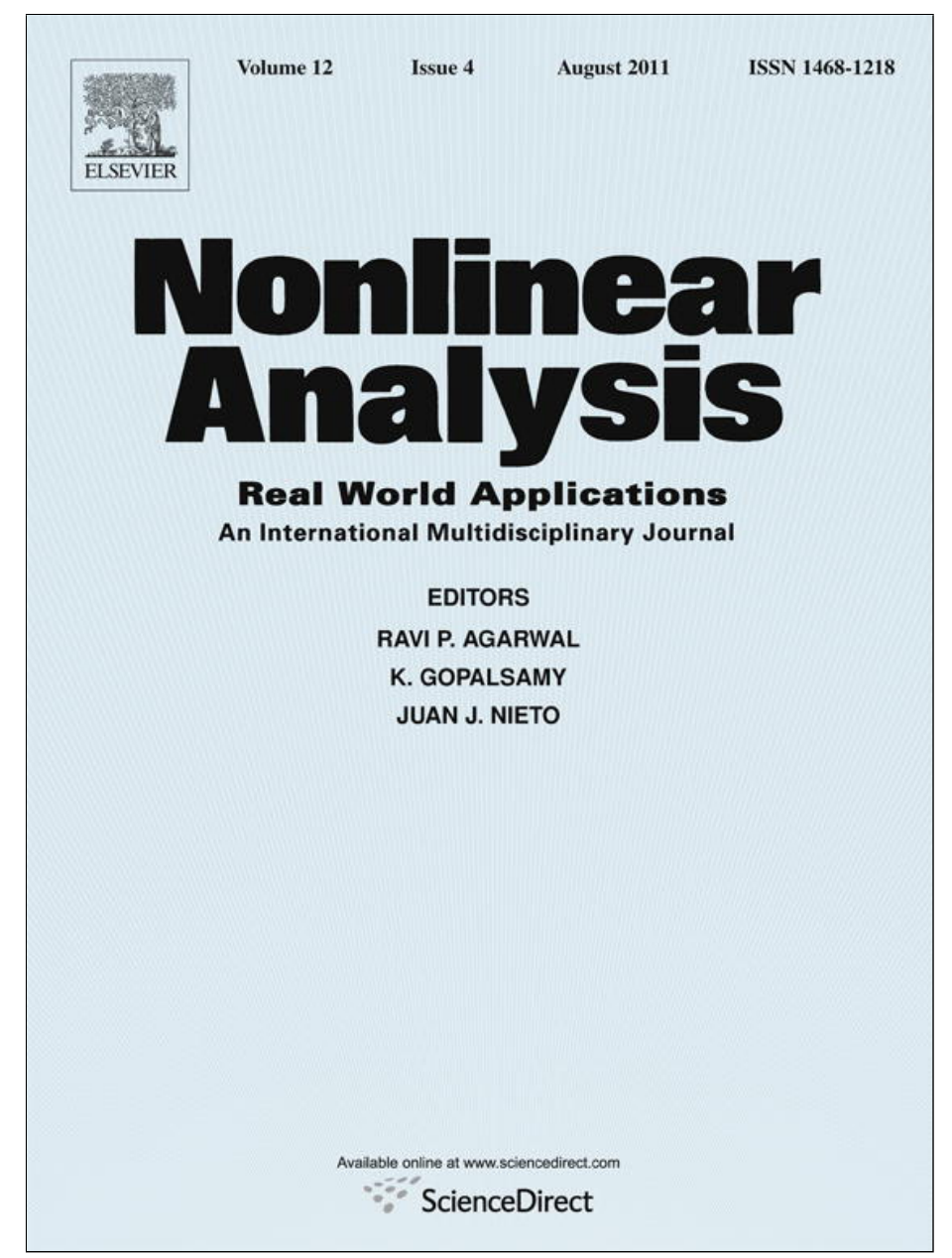

This article appeared in a journal published by Elsevier. The attached copy is furnished to the author for internal non-commercial research and education use, including for instruction at the authors institution and sharing with colleagues.

Other uses, including reproduction and distribution, or selling or licensing copies, or posting to personal, institutional or third party websites are prohibited.

In most cases authors are permitted to post their version of the article (e.g. in Word or Tex form) to their personal website or institutional repository. Authors requiring further information regarding Elsevier's archiving and manuscript policies are encouraged to visit:

http://www.elsevier.com/copyright 


\title{
Permanence and global stability of a class of discrete epidemic models
}

\author{
Yoshiaki Muroya ${ }^{\mathrm{a}, *}$, Yukihiko Nakata $^{\mathrm{b}}$, Giuseppe $\operatorname{Izzo}^{\mathrm{c}}$, Antonia Vecchio ${ }^{\mathrm{d}}$ \\ a Department of Mathematical Sciences, Waseda University, 3-4-1 Ohkubo Shinjuku-ku, Tokyo, 169-8555, Japan \\ b BCAM - Basque Center for Applied Mathematics, Bizkaia Technology Park, Building 500, E-48160, Derio, Spain \\ ${ }^{\mathrm{c}}$ Dipartimento di Matematica e Applicazioni, “R. Caccioppoli”, Università degli studi di Napoli "Federico II”, Via Cintia, Monte S. Angelo, I-80126 Napoli, Italy \\ d Ist. per le Appl. del Calcolo “M. Picone”, Sede di Napoli, CNR, Via P. Castellino, 111, 80131 Napoli, Italy
}

\section{A R T I C L E I N F O}

\section{Article history}

Received 20 June 2010

Accepted 29 December 2010

\section{Keywords:}

Discrete epidemic model

Permanence

Global asymptotic stability

Endemic equilibrium

\begin{abstract}
A B S T R A C T
In this paper we investigate the permanence of a system and give a sufficient condition for the endemic equilibrium to be globally asymptotically stable, which are the remaining problems in our previous paper (G. Izzo, Y. Muroya, A. Vecchio, A general discrete time model of population dynamics in the presence of an infection, Discrete Dyn. Nat. Soc. (2009), Article ID 143019, 15 pages. doi:10.1155/2009/143019.)
\end{abstract}

(c) 2011 Elsevier Ltd. All rights reserved.

\section{Introduction}

The application of theories of functional differential/difference equations in mathematical biology has been developing rapidly. Various mathematical models have been proposed in the literature of population dynamics, ecology and epidemiology. Many authors have studied the dynamical behavior of several epidemic models (see [1-21] and the references therein).

For a discrete epidemic model with immigration of infectives, Jang and Elaydi [10] showed the global asymptotic stability of the disease-free equilibrium, the local asymptotic stability of the endemic equilibrium and the strong persistence of the susceptible class by means of the nonstandard discretization method.

Applying the techniques offered in [20] to discrete models, Sekiguchi [15] and Sekiguchi and Ishiwata [16] proved the permanence of a class of SIR discrete epidemic models with one delay and SEIRS discrete epidemic models with two delays if an endemic equilibrium of each model exists.

In those cases, how to choose the discrete schemes which guarantee the global asymptotic stability for the endemic equilibrium of the models still remained a very important open problem. A first complete answer to this problem has been established by the recent paper [3].

Recently, extending the result in [5], Yuan and Zou [21], proposed a general mathematical model with detailed justifications to describe the spread of a disease with latency in a heterogeneous host population which includes many existing ones as special cases (see [18] for more detail). In a particular case, one group of this model becomes the following model for a disease with a latent period:

\footnotetext{
* Corresponding author.

E-mail addresses: ymuroya@waseda.jp (Y. Muroya), nakata@bcamath.org (Y. Nakata), giuseppe.izzo@unina.it (G. Izzo), a.vecchio@na.iac.cnr.it (A. Vecchio).
} 


$$
\left\{\begin{array}{l}
S^{\prime}(t)=\varphi(S)-\beta S(t) I(t), \\
I^{\prime}(t)=\beta \int_{0}^{t} S(u) I(u) P(t-u) \mathrm{e}^{-\delta(t-u)} \mathrm{d} u-(\delta+\epsilon+\gamma) I(t), \\
R^{\prime}(t)=\gamma I(t)-\delta R(t) .
\end{array}\right.
$$

$S(t), I(t)$ and $R(t)$ denote the numbers of susceptible, infectious, and recovered individuals at time $t$, respectively. The nonnegative constant $\beta$ is the transmission rate due to the contact of susceptible individuals with infectious individuals. The nonnegative constants $\delta, \epsilon, \gamma$ are natural death rates, disease-caused death rates and recovery rates, respectively. The function $P(t)$ is the gamma distribution:

$$
P(u)=P_{n, b}(u) \equiv \frac{u^{n-1}}{(n-1) ! b^{n}} \mathrm{e}^{-u / b},
$$

where $b>0$ is a real number and $n>1$ is an integer. $\varphi$ is a $C^{1}$ non-increasing function and there exists $S^{0}$ such that

$$
\varphi\left(S^{0}\right)=0, \quad \varphi(u)>0, \quad \text { for } 0 \leq u<S^{0}, \quad \text { and } \varphi(u)<0 \text { for } u>S^{0} .
$$

By using the "linear chain trick" to transfer (1) into a system of ordinary differential equations, Yuan and Zou [21] derived the following model which is equivalent to the integro-differential system (1):

$$
\left\{\begin{array}{l}
S^{\prime}(t)=\varphi(S(t))-\beta S(t) I(t) \\
y_{1}^{\prime}(t)=c(S(t)) y_{n+1}(t)-d y_{1}(t) \\
y_{j}^{\prime}(t)=d y_{1}(t)-d y_{2}(t), \quad j=2,3, \ldots, n, \\
y_{n+1}^{\prime}(t)=d y_{n}(t)-e y_{n+1}(t)
\end{array}\right.
$$

where

$$
\left\{\begin{array}{l}
c(S)=\frac{\beta S}{(1+\delta b)^{n}}, \quad d=\frac{1}{\hat{b}}, \quad e=\delta+\epsilon+\gamma . \\
\hat{b}=\frac{b}{1+\delta b}, \quad y_{n+1}(t)=I(t) .
\end{array}\right.
$$

The reproduction number of system (4) becomes

$$
R_{0}=\frac{c\left(S^{0}\right)}{e}=\frac{\beta S^{0}}{(1+\delta b)^{n}(\delta+\epsilon+\gamma)} .
$$

Yuan and Zou [21] established a complete analysis of the global asymptotic stability of system (4) with a single threshold parameter $R_{0}$ : if $R_{0} \leq 1$, the disease-free equilibrium is globally asymptotically stable in the positive orthant, whereas if $R_{0}>1$, a unique endemic equilibrium exists and is globally asymptotically stable in the interior of the positive orthant. as a special case (one group) of multi-group cases.

For this result of a continuous model, Muroya et al. [13] propose the following discrete SIR epidemic model which is derived from system (4) by applying a variation of the backward Euler method (cf. [9]):

$$
\left\{\begin{array}{l}
s(p+1)=s(p)+\varphi(s(p+1))-\beta s(p+1) y_{n+1}(p) \\
y_{1}(p+1)=y_{1}(p)+c(s(p+1)) y_{n+1}(p)-d y_{1}(p+1), \\
y_{j}(p+1)=y_{j}(p)+d y_{j-1}(p+1)-d y_{j}(p+1), j=2,3, \ldots, n \\
y_{n+1}(p+1)=y_{n+1}(p)+d y_{n}(p+1)-e y_{n+1}(p+1)
\end{array}\right.
$$

where the initial condition of system $(7)$ is

$$
s(0)>0, \quad y_{j}(p)>0, \quad \text { and } j=1, \ldots, n+1 .
$$

Using the same threshold $R_{0}=\frac{c\left(S^{0}\right)}{e}=\frac{\beta S^{0}}{(1+\delta b)^{n}(\delta+\epsilon+\gamma)}$ as the continuous system (4) and applying new techniques to both cases for $R_{0} \leq 1$ and $R_{0}>1$, they established a complete analysis of the global asymptotic stability for this discrete SIR epidemic model ( 7 ) with latency spreading in a heterogeneous host population. In particular, they extended both techniques in [8,9] for the case $R_{0} \leq 1$ and a simpler proof in [3] for the permanence of system (7) than Sekiguchi [15] and Sekiguchi and Ishiwata [16] for system (7) and they also applied techniques of Lyapunov functions in [11] to prove the global asymptotic stability for the endemic equilibrium of system (7) for the case $R_{0}>1$.

On the other hand, Izzo et al. [8] presented a set of difference equations

$$
\left\{\begin{array}{l}
y(n+1)=\alpha+(1-\beta) y(n)-\sum_{i=1}^{m} \psi_{i}(y(n+1)) z_{i}(n+1), \quad n \geq 0, \\
z_{1}(n+1)=\left(1-a_{1}\right) z_{1}(n)+\phi_{1}(y(n)) z_{L}(n), \quad 1 \leq L \leq m, n \geq 0, \\
z_{i}(n+1)=\left(1-a_{i}\right) z_{i}(n)+\phi_{i}(y(n)) z_{i-1}(n), \quad i=2,3, \ldots, m, n \geq 0,
\end{array}\right.
$$

which generalizes that proposed in [9] and represented the discrete counterpart of a larger class of continuous model concerning the dynamics of an infection in an organism or in a host population. This difference system contains a very large class of population dynamics models in the presence of an infection involving typically at least two populations: susceptible 
individuals and infective ones. The former is represented in (9) by the sequence $y$, while the latter is represented by the sequences $z_{i}$. In [8], various examples of continuous models which can be discretized by (9) were reported.

Izzo et al. [8] proved some basic properties of the solution of the proposed scheme such as positivity and boundedness which makes it meaningful in the applications, and investigated the asymptotic behavior of the solution and established a necessary and sufficient condition for the vanishing of the sequences of the infection and derived the expression of the basic reproduction number $R_{0}$, a threshold parameter which allows us to predict whether the infection develops or not. But for the case $R_{0}>1$, they only prove the existence of endemic equilibrium and open questions remain about the permanence and the global asymptotic stability for the endemic equilibrium of the system.

Motivated by the above results, in this paper, we assume that

$\psi_{i}(y)$ is a monotone increasing function of $y, \quad 1 \leq i \leq m$,

and we prove the permanence of the system (9), and give a partial answer to the remaining open question for the case $R_{0}>1$ about the global asymptotic stability for the endemic equilibrium of the system (9). To this aim, we consider the following modified difference equations to (9):

$$
\left\{\begin{array}{l}
y(n+1)=\alpha+(1-\beta) y(n)-\sum_{i=1}^{m} \psi_{i}(y(n+1)) x_{i}(n), \quad n \geq 0, \\
x_{1}(n+1)=\left(1-a_{1}\right) x_{1}(n)+\phi_{1}(y(n+1)) x_{L}(n), \quad 1 \leq L \leq m, \\
x_{i}(n+1)=\left(1-a_{i}\right) x_{i}(n)+\phi_{i}(y(n+1)) x_{i-1}(n), \quad i=2,3, \ldots, m
\end{array}\right.
$$

where $\psi_{i}(x), \phi_{i}(x) \in C^{0}(\mathbb{R}), 1 \leq i \leq m$ and we assume that

(i) $\alpha>0$,

(ii) $0<\beta<1$,

(iii) $0<a_{i}<1, i=1,2, \ldots, m$,

(iv) $\phi_{i}(y)$ is strictly monotone increasing and $\phi_{i}(0) \geq 0, i=1, \ldots, m$,

(v) $y \psi_{i}(y) \geq 0, \psi_{i}(0)=0, \forall y \in \mathbb{R}, i=1,2, \ldots, m$,

(vi) $\exists \bar{i}$ s.t. $1 \leq \bar{i} \leq L$ and

$$
\exists q>0: \begin{cases}\phi_{1}(y) \leq q \psi_{L}(y), & y \geq 0 \text { if } \bar{i}=1 \\ \phi_{\bar{i}}(y) \leq q \psi_{\bar{i}-1}(y), & y \geq 0 \text { if } 2 \leq \bar{i} \leq L .\end{cases}
$$

Note that (10) is equivalent to the following difference equations:

$$
\left\{\begin{array}{l}
y(n+1)-y(n)=\frac{1}{1-\beta}\left\{\alpha-\beta y(n+1)-\sum_{i=1}^{m} \psi_{i}(y(n+1)) x_{i}(n)\right\}, \\
x_{1}(n+1)-x_{1}(n)=\frac{1}{1-a_{1}}\left\{-a_{1} x_{1}(n+1)+\phi_{1}(y(n+1)) x_{L}(n)\right\}, \quad 1 \leq L \leq m, \\
x_{i}(n+1)-x_{i}(n)=\frac{1}{1-a_{i}}\left\{-a_{i} x_{i}(n+1)+\phi_{i}(y(n+1)) x_{i-1}(n)\right\}, \quad i=2,3, \ldots, m, n \geq 0,
\end{array}\right.
$$

and if we set $z_{i}(n+1)=x_{i}(n), i=1,2, \ldots, m, n=0,1, \ldots$, then (10) becomes the set of difference equations (9) except for initial equations of $n=0$ for $z_{i}(1), i=1,2, \ldots, m$, that is, equations of

$$
\begin{cases}z_{1}(1)=\left(1-a_{1}\right) z_{1}(0)+\phi_{1}(y(1)) z_{L}(0), & 1 \leq L \leq m, n \geq 1 \\ z_{i}(1)=\left(1-a_{i}\right) z_{i}(0)+\phi_{i}(y(0)) z_{i-1}(0), & i=2,3, \ldots, m, n \geq 1 .\end{cases}
$$

Thus, the asymptotic behaviors of the solutions of (10) and the difference equations (9) treated in Izzo et al. [8] are the same.

The paper is organized as follows. In Section 2, we prove some basic properties of the solution of the proposed scheme such as positivity and eventual boundedness which do not depend on the initial conditions. In Section 3, the asymptotic behavior of the solution is investigated. We prove a necessary and sufficient condition for the vanishing of the sequences $\left\{x_{i}(n)\right\}$ and we derive the expression of the basic reproduction number, a threshold parameter which allows us to predict whether the infection develops or not. Such a parameter permits us to check that the asymptotic behaviors of the discrete and continuous problems coincide, therefore our discrete system incorporates the dynamical characteristics (such as positivity and steady states) of the continuous-time models. In Section 4, we prove the permanence of system (10), and in Section 5, for a class of special models of (10), we show that the endemic equilibrium is globally asymptotically stable. Finally, we offer a short conclusion in Section 6.

\section{Basic properties}

Since the functions $y$ and $x_{i}(i=1,2, \ldots, m)$ represent populations, at first, we can prove in the following two theorems their positivity and eventual boundedness which do not depend on the initial conditions. 
Lemma 2.1. Under the conditions (i)-(v), it holds that $y(n)>0, x_{i}(n)>0 \quad n \geq 0, i=1, \ldots, m$.

Proof. Suppose that

$$
y(1) \leq 0 \text {. }
$$

From (v) and $x_{i}(0)>0, i=1,2, \ldots, m$, we get $\sum_{i=1}^{m} \psi_{i}(y(1)) x_{i}(0) \leq 0$, and from (i), (ii) and the first of (10) we obtain $y(1)>0$ which contradicts (13). Then, from (iii), (iv) and the positivity of $x_{i}(0)$ we have $x_{i}(1)>0, i=1,2, \ldots, m$. The rest of this lemma can be proved in the same way (by induction).

Put

$$
V(n) \equiv y(n)+\sum_{i=1}^{m} \psi(y(n)) x_{i}(n-1), \quad n=1,2, \ldots
$$

Lemma 2.2. It holds that

$$
\limsup _{n \rightarrow \infty} V(n) \leq \frac{\alpha}{\beta} \text {. }
$$

Proof. By (10) and $y(n) \leq V(n)$ for $n=1,2, \ldots$, we have that

$$
V(n+1)=y(n+1)+\sum_{i=1}^{m} \psi(y(n+1)) x_{i}(n)=\alpha+(1-\beta) y(n) \leq \alpha+(1-\beta) V(n),
$$

and

$$
V(n+1)-V(n) \leq \frac{1}{1-\beta}\{\alpha-\beta V(n+1)\},
$$

from which we obtain the conclusion of this lemma.

In order to simplify the proofs of the remaining section, let us set

$$
\left\{\begin{array}{l}
\underline{y}=\liminf _{n \rightarrow \infty} y(n), \quad \bar{y}=\limsup _{n \rightarrow \infty} y(n), \\
x_{0}(n)=x_{L}(n), \quad n=0,1,2, \ldots, \\
\underline{x}_{i}=\liminf _{n \rightarrow \infty} x_{i}(n), \quad \bar{x}_{i}=\limsup _{n \rightarrow \infty} x_{i}(n), \quad 1 \leq i \leq m,
\end{array}\right.
$$

and introduce the following basic lemma of the eventual boundedness of $y(n)$ and $x_{i}(n), 1 \leq i \leq m$ by Lemma 2.2 (cf. Izzo et al. [8, Theorem 3.2]). The eventual boundedness of $x(n), n=0,1,2, \ldots$ means that there exists a constant $\bar{x}$ such that $\bar{x}$ is independent of the initial conditions and $x(n) \leq \bar{x}$ for any sufficiently large $t$.

Lemma 2.3. Under the conditions (i)-(vi), it holds that

$$
\bar{y} \leq \frac{\alpha}{\beta}
$$

and for $\bar{i}=1$,

$$
\left\{\begin{array}{l}
\bar{x}_{1} \leq \frac{q \alpha}{a_{1} \beta}, \quad \bar{x}_{2} \leq \frac{q \alpha}{a_{1} \beta} \frac{\phi_{2}\left(\frac{\alpha}{\beta}\right)}{a_{2}}, \quad \bar{x}_{3} \leq \frac{q \alpha}{a_{1} \beta} \frac{\phi_{2}\left(\frac{\alpha}{\beta}\right)}{a_{2}} \frac{\phi_{3}\left(\frac{\alpha}{\beta}\right)}{a_{3}}, \ldots, \\
\bar{x}_{m} \leq \frac{q \alpha}{a_{1} \beta} \frac{\phi_{2}\left(\frac{\alpha}{\beta}\right)}{a_{2}} \frac{\phi_{3}\left(\frac{\alpha}{\beta}\right)}{a_{3}} \cdots \frac{\phi_{m}\left(\frac{\alpha}{\beta}\right)}{a_{m}},
\end{array}\right.
$$

and for $2 \leq \bar{i} \leq m$,

$$
\left\{\begin{array}{l}
\bar{x}_{i} \leq \frac{q \alpha}{a_{i} \beta} \frac{\phi_{\bar{i}+1}\left(\frac{\alpha}{\beta}\right)}{a_{\bar{i}+1}} \frac{\phi_{\bar{i}+2}\left(\frac{\alpha}{\beta}\right)}{a_{\bar{i}+2}} \cdots \frac{\phi_{L}\left(\frac{\alpha}{\beta}\right)}{a_{L}} \frac{\phi_{1}\left(\frac{\alpha}{\beta}\right)}{a_{1}} \cdots \frac{\phi_{i}\left(\frac{\alpha}{\beta}\right)}{a_{i}}, \quad 1 \leq i \leq \bar{i}-1, \\
\bar{x}_{\bar{i}} \leq \frac{q \alpha}{a_{\bar{i}} \beta}, \quad \bar{x}_{\bar{i}+1} \leq \frac{q \alpha}{a_{\bar{i}} \beta} \frac{\phi_{\bar{i}+1}\left(\frac{\alpha}{\beta}\right)}{a_{\bar{i}+1}}, \ldots, \bar{x}_{L} \leq \frac{q \alpha}{a_{\bar{i}} \beta} \frac{\phi_{\bar{i}+1}\left(\frac{\alpha}{\beta}\right)}{a_{\bar{i}+1}} \frac{\phi_{\bar{i}+2}\left(\frac{\alpha}{\beta}\right)}{a_{\bar{i}+2}} \cdots \frac{\phi_{L}\left(\frac{\alpha}{\beta}\right)}{a_{L}}, \\
\bar{x}_{i} \leq \frac{q \alpha}{a_{\bar{i}} \beta} \frac{\phi_{\bar{i}+1}\left(\frac{\alpha}{\beta}\right)}{a_{\bar{i}+1}} \frac{\phi_{\bar{i}+2}\left(\frac{\alpha}{\beta}\right)}{a_{\bar{i}+2}} \cdots \frac{\phi_{i}\left(\frac{\alpha}{\beta}\right)}{a_{i}}, \quad L+1 \leq i \leq m,
\end{array}\right.
$$

and the sequences $\{y(n)\},\left\{x_{i}(n)\right\}, i=1,2, \ldots, m$ are eventually bounded. 
Proof. Since the hypotheses of the previous lemma hold, positivity of the sequences $\{y(n)\},\left\{x_{i}(n)\right\}$ is assured. Thus, by Lemma 2.2, we easily obtain that $\bar{y} \leq \limsup _{n \rightarrow \infty} V(n) \leq \frac{\alpha}{\beta}$, and $\limsup _{n \rightarrow \infty} \phi_{i}(y(n)) x_{i-1}(n-1) \leq \limsup _{n \rightarrow \infty}$ $\phi_{i}\left(\frac{\alpha}{\beta}\right) x_{i-1}(n-1) \leq \frac{\alpha}{\beta}, 1 \leq i \leq L$. Assume that there exists $q>0$ such that

$$
\phi_{1}(y) \leq q \psi_{L}(y), \quad y \geq 0 .
$$

Then, $\bar{i}=1$ and in order to prove this theorem it is convenient to represent (10) in the form of the following system of Volterra difference equations (see for example [1,2]), we have:

$$
\begin{aligned}
x_{1}(n+1) & =\left(1-a_{1}\right)^{n+1} x_{1}(0)+\sum_{l=1}^{n+1}\left(1-a_{1}\right)^{n+1-l} \phi_{1}(y(l)) x_{L}(l-1), \\
& \leq\left(1-a_{1}\right)^{n+1} x_{1}(0)+q \sum_{l=1}^{n+1}\left(1-a_{1}\right)^{n+1-l} \psi_{L}(y(l)) x_{L}(l-1) .
\end{aligned}
$$

Then, we have that

$$
\bar{x}_{1}=\limsup _{n \rightarrow \infty} x_{1}(n) \leq q \frac{\limsup _{n \rightarrow \infty} \psi_{L}\left(\frac{\alpha}{\beta}\right) x_{L}(n-1)}{a_{1}} \leq q \frac{\frac{\alpha}{\beta}}{a_{1}}=\frac{q \alpha}{\beta a_{1}} .
$$

Let us consider the third of (10) for $i=2$. Then, similarly, we have that

$$
x_{2}(n+1) \leq\left(1-a_{2}\right)^{n+1} x_{2}(0)+\sum_{l=1}^{n+1}\left(1-a_{2}\right)^{n+1-l} \phi_{2}(y(l)) x_{1}(l-1) .
$$

From the eventual boundedness of $x_{1}(n)$ and (iv) we have:

$$
\bar{x}_{2}=\limsup _{n \rightarrow \infty} x_{2}(n) \leq \frac{\psi_{2}\left(\frac{\alpha}{\beta}\right) \bar{x}_{1}}{a_{2}} \leq \frac{q \alpha}{a_{1} \beta} \frac{\phi_{2}\left(\frac{\alpha}{\beta}\right)}{a_{2}} .
$$

The eventual boundedness of the remaining sequences can be proved in the same way and we obtain (18). If (20) does not hold then, from (vi), there exist $q>0$ and $\bar{i}$ such that $2 \leq \bar{i} \leq L$ and

$$
\phi_{\bar{i}}(y) \leq q \psi_{\bar{i}-1}(y), \quad y \geq 0 .
$$

Thus, by the third of (10), (ii), (iii) and (11), the eventual boundedness of $x_{\bar{i}}(n)$ (and then the $x_{j}(n)$ sequences, $j>\bar{i}$ ) can be proved with the same argumentation used before for $x_{1}(n)$ and $x_{2}(n)$. Since by $2 \leq \bar{i} \leq L, x_{i}(n), \bar{i} \leq i \leq m$ is eventually bounded, we obtain the eventual boundedness of the remaining sequences $x_{j}(n), 1 \leq j \leq \bar{i}$ and obtain (19).

Similar to Izzo et al. [8, Lemma 2.3]), we obtain the following lemma which only improves the lower and upper bounds of $y$ and $\bar{y}$ compared with those of Izzo et al. [8, Lemma 2.3].

\section{Lemma 2.4.}

$$
\begin{aligned}
& 0<h^{-1}\left(\alpha ; \bar{x}_{1}, \bar{x}_{2}, \ldots, \bar{x}_{m}\right) \leq \underline{y} \leq \bar{y} \leq h^{-1}\left(\alpha ; \underline{x}_{1}, \underline{x}_{2}, \ldots, \underline{x}_{m}\right) \leq \frac{\alpha}{\beta}, \\
& \frac{\phi_{i}(\underline{y})}{a_{i}} \underline{x}_{i-1} \leq \underline{x}_{i} \leq \bar{x}_{i} \leq \frac{\phi_{i}(\bar{y})}{a_{i}} \bar{x}_{i-1}, \quad i=1,2, \ldots, m, \\
& \underline{x}_{i} \geq\left(\prod_{j=1}^{L} \frac{\phi_{j}(\underline{y})}{a_{j}}\right) \underline{x}_{i}, \quad \text { and } \\
& \bar{x}_{i} \leq\left(\prod_{j=1}^{L} \frac{\phi_{j}(\bar{y})}{a_{j}}\right) \bar{x}_{i}, \quad i=1,2, \ldots, L,
\end{aligned}
$$

where for any fixed nonnegative constants $x_{1}, x_{2}, \ldots, x_{m}$,

$$
h\left(y ; x_{1}, x_{2}, \ldots, x_{m}\right) \equiv \beta y+\sum_{i=1}^{m} \psi_{i}(y) x_{i},
$$

is a strictly monotone increasing function of $y$, and for a positive constant $z$, there exists the inverse function $y=$ $h^{-1}\left(z ; x_{1}, x_{2}, \ldots, x_{m}\right)$ of $z=h\left(y ; x_{1}, x_{2}, \ldots, x_{m}\right)$.

Proof. By the assumption that each $\psi_{i}(y)$ is a monotone increasing function of $y, 1 \leq i \leq m, h^{-1}\left(y ; x_{1}, x_{2}, \ldots, x_{m}\right)$ is a strictly monotone increasing function of $y$. From Eq. (10) and Lemmas 2.1 and 2.3, we easily have that 


$$
\underline{y} \geq \alpha+(1-\beta) \underline{y}-\sum_{i=1}^{m} \psi_{i}(\underline{y}) \bar{x}_{i}
$$

and then

$$
\beta \underline{y}+\sum_{i=1}^{m} \psi_{i}(\underline{y}) \bar{x}_{i} \geq \alpha .
$$

Thus, we obtain that $y \geq h^{-1}\left(\alpha ; \bar{x}_{1}, \bar{x}_{2}, \ldots, \bar{x}_{m}\right)$. Since for any fixed nonnegative constants $x_{1}, x_{2}, \ldots, x_{m}$, $h^{-1}\left(0 ; x_{1}, x_{2}, \ldots, x_{m}\right)=0$ and $h^{-1}\left(y ; x_{1}, x_{2}, \ldots, x_{m}\right)$ is a strictly monotone increasing function of $y$, by $\alpha>0$, we have that

$$
0<h^{-1}\left(\alpha ; \bar{x}_{1}, \bar{x}_{2}, \ldots, \bar{x}_{m}\right) \leq \underline{y} .
$$

In the same way it can be proved that

$$
\bar{y} \leq h^{-1}\left(\alpha ; \underline{x}_{1}, \underline{x}_{2}, \ldots, \underline{x}_{m}\right) \leq \frac{\alpha}{\beta} .
$$

Also, from (11), we easily have that

$$
\frac{\phi_{i}(\underline{y})}{a_{i}} \underline{x}_{i-1} \leq \underline{x}_{i} \leq \bar{x}_{i} \leq \frac{\phi_{i}(\bar{y})}{a_{i}} \bar{x}_{i-1}, \quad i=1,2, \ldots, m,
$$

and we have that

$$
\begin{aligned}
\bar{x}_{L} & \leq\left(\prod_{j=1}^{L} \frac{\phi_{j}(\bar{y})}{a_{j}}\right) \bar{x}_{L}, \\
\bar{x}_{i} & \leq \frac{\phi_{i}(\bar{y})}{a_{i}} \bar{x}_{i-1} \leq \cdots \leq\left(\prod_{j=2}^{i} \frac{\phi_{j}(\bar{y})}{a_{j}}\right) \bar{x}_{1} \leq\left(\prod_{j=1}^{i} \frac{\phi_{j}(\bar{y})}{a_{j}}\right) \bar{x}_{L} \\
& \leq\left(\prod_{j=1}^{i} \frac{\phi_{j}(\bar{y})}{a_{j}}\right) \frac{\phi_{L}(\bar{y})}{a_{L}} \bar{x}_{L-1} \leq \cdots \leq\left(\prod_{j=1}^{i} \frac{\phi_{j}(\bar{y})}{a_{j}}\right)\left(\prod_{j=i+1}^{L} \frac{\phi_{j}(\bar{y})}{a_{j}}\right) \bar{x}_{i} \\
& =\left(\prod_{j=1}^{L} \frac{\phi_{j}(\bar{y})}{a_{j}}\right) \bar{x}_{i}, \quad 1 \leq i \leq L
\end{aligned}
$$

and then

$$
\bar{x}_{i} \leq\left(\prod_{j=1}^{L} \frac{\phi_{j}(\bar{y})}{a_{j}}\right) \bar{x}_{i}, \quad 1 \leq i \leq L .
$$

Similarly, we have that

$$
\underline{x}_{i} \geq\left(\prod_{j=1}^{L} \frac{\phi_{j}(\underline{y})}{a_{j}}\right) \underline{x}_{i}, \quad 1 \leq i \leq L .
$$

The remaining parts are obtained similarly.

Note that if there exists an integer $i \in\{1,2, \ldots, m\}$ such that $\underline{x}_{i}>0$, then by the last inequalities of (26) in Lemma 2.4 , we have that

$$
\prod_{j=1}^{L} \frac{\phi_{j}(\underline{y})}{a_{j}} \leq 1 \leq \prod_{j=1}^{L} \frac{\phi_{j}(\bar{y})}{a_{j}} .
$$

\section{Asymptotic properties}

By hypotheses (iii)-(v), it holds that

$$
\text { (vii) } \begin{aligned}
P(\lambda) \equiv & \prod_{j=1}^{L} \frac{\phi_{j}(\lambda)}{a_{j}} \text { is a strictly increasing positive } \\
& \text { continuous function of } \lambda \text { on }(0,+\infty) \text { and } P(0)=0<1,
\end{aligned}
$$


and we put

$$
R_{0}=P\left(\frac{\alpha}{\beta}\right) .
$$

Since the asymptotic behaviors of the solutions of (10) and the difference equations (9) treated in Izzo et al. [8] are exactly the same, as mentioned in Section 1, we obtain Theorem 3.1 by Izzo et al. [8].

Theorem 3.1. (i) $R_{0} \leq 1$, if and only if, the disease free equilibrium $E_{0}=\left(\frac{\alpha}{\beta}, 0,0, \ldots, 0\right)$ of (1) is globally asymptotically stable and hence

$$
\lim _{n \rightarrow \infty} y(n)=\frac{\alpha}{\beta} \quad \text { and } \quad \lim _{n \rightarrow \infty} x_{i}(n)=0, \quad i=1,2, \ldots, m .
$$

(ii) $R_{0}>1$, if and only if,

$$
\bar{y}<\frac{\alpha}{\beta} \quad \text { and } \quad \underline{x}_{i}>0, \quad 1 \leq i \leq m,
$$

and there exists the endemic equilibrium $E^{*}=\left(y^{*}, x_{1}^{*}, x_{2}^{*}, \ldots, x_{m}^{*}\right)$ of (1) such that

$$
\left\{\begin{array}{l}
P\left(y^{*}\right)=1, \quad 0<y^{*}<\frac{\alpha}{\beta}, \quad \phi_{i}\left(y^{*}\right) x_{i-1}^{*}=a_{i} x_{i}^{*}, \quad i=1,2, \ldots, m, \\
\text { and } x_{i}^{*}=\frac{\alpha-\beta y^{*}}{\sum_{l=1}^{m} \psi_{l}\left(y^{*}\right) \prod_{j=i+1}^{l} \frac{\phi_{j}\left(y^{*}\right)}{a_{j}}}>0, \quad i=1,2, \ldots, m .
\end{array}\right.
$$

\section{Global stability of the endemic equilibrium for $R_{0}>1$}

In this section, we assume that $R_{0}>1$ and we obtain the permanence of system (10) for $R_{0}>1$.

It holds that

$$
\prod_{i=1}^{m} \frac{\phi_{i}\left(y^{*}\right)}{a_{i}}=1 .
$$

We have a lemma.

Lemma 4.1. For $R_{0}>1$, it holds that $x_{i}^{*}>0, i=1,2, \ldots, m$ and

$$
\frac{\phi_{i}\left(y^{*}\right) x_{i-1}^{*}}{a_{i} x_{i}^{*}}=1, \quad 1 \leq i \leq m .
$$

Proof. From (35), we obtain (37).

By Lemma 4.1, we put

$$
\left\{\begin{array}{l}
\tilde{\psi}_{i}(y)=\psi_{i}(y) x_{i}^{*}, \quad \tilde{\phi}_{i}(y)=\frac{\phi_{i}(y) x_{i-1}^{*}}{x_{i}^{*}} \\
\tilde{x}_{i}(n)=\frac{x_{i}(n)}{x_{i}^{*}}, \quad \tilde{x}_{i}^{*}=1, \quad 1 \leq i \leq m, n=0,1,2, \ldots
\end{array}\right.
$$

Then, (11) becomes that

$$
\left\{\begin{array}{l}
y(n+1)-y(n)=\frac{1}{1-\beta}\left\{\alpha-\beta y(n+1)-\sum_{i=1}^{m} \tilde{\psi}_{i}(y(n+1)) \tilde{x}_{i}(n)\right\}, \\
\tilde{x}_{1}(n+1)-\tilde{x}_{1}(n)=\frac{1}{1-a_{1}}\left\{-a_{1} \tilde{x}_{1}(n+1)+\tilde{\phi}_{1}(y(n+1)) \tilde{x}_{L}(n)\right\}, \quad 1 \leq L \leq m, \\
\tilde{x}_{i}(n+1)-\tilde{x}_{i}(n)=\frac{1}{1-a_{i}}\left\{-a_{i} \tilde{x}_{i}(n+1)+\tilde{\phi}_{i}(y(n+1)) \tilde{x}_{i-1}(n)\right\}, \quad i=2,3, \ldots, m, n \geq 0,
\end{array}\right.
$$

and by Lemma 4.1, it holds that

$$
\frac{\tilde{\phi}_{i}\left(y^{*}\right)}{a_{i}}=1, \quad 1 \leq i \leq m .
$$

Thus, (10) becomes that 


$$
\left\{\begin{array}{l}
y(n+1)-y(n)=\frac{1}{1-\beta}\left\{\alpha-\beta y(n+1)-\sum_{i=1}^{m} \tilde{\psi}_{i}(y(n+1)) \tilde{x}_{i}(n)\right\}, \\
\tilde{x}_{1}(n+1)-\tilde{x}_{1}(n)=\frac{a_{1}}{1-a_{1}}\left\{\frac{\tilde{\phi}_{1}(y(n+1))}{\tilde{\phi}_{1}\left(y^{*}\right)} \tilde{x}_{L}(n)-\tilde{x}_{1}(n+1)\right\}, \quad 1 \leq L \leq m, \\
\tilde{x}_{i}(n+1)-\tilde{x}_{i}(n)=\frac{a_{i}}{1-a_{i}}\left\{\frac{\tilde{\phi}_{i}(y(n+1))}{\tilde{\phi}_{i}\left(y^{*}\right)} \tilde{x}_{i-1}(n)-\tilde{x}_{i}(n+1)\right\}, \\
i=2,3, \ldots, m, n \geq 0 .
\end{array}\right.
$$

Lemma 4.2. If $\min _{1 \leq i \leq m} \tilde{x}_{i}(n+1)<\min _{1 \leq i \leq m} \tilde{x}_{i}(n)$, then $y(n+1)<y^{*}$. Inversely, if $y(n+1) \geq y^{*}$, then $\min _{1 \leq i \leq m} \tilde{x}_{i}(n+1) \geq$ $\min _{1 \leq i \leq m} \tilde{x}_{i}(n)$.

Proof. Assume that there exists a positive integer $1 \leq i_{0} \leq m$ such that $0<\tilde{x}_{i_{0}}(n+1)=\min _{1 \leq i \leq m} \tilde{x}_{i}(n+1)<$ $\min _{1 \leq i \leq m} \tilde{x}_{i}(n)$. Then, $\tilde{x}_{i_{0}}(n+1)-\tilde{x}_{i_{0}}(n)<0$ and $\tilde{x}_{i_{0}}(n+1)-\tilde{x}_{i_{0}-1}(n)<0$, and by $(41)$, we have that

$$
0>\tilde{x}_{i_{0}}(n+1)-\tilde{x}_{i_{0}}(n) \geq \frac{a_{i_{0}}}{1-a_{i_{0}}}\left\{\frac{\tilde{\phi}_{i_{0}}(y(n+1))}{\tilde{\phi}_{i_{0}}\left(y^{*}\right)}-1\right\} \tilde{x}_{i_{0}}(n+1),
$$

from which we obtain that $\tilde{\phi}_{i_{0}}(y(n+1))<\tilde{\phi}_{i_{0}}\left(y^{*}\right)$. Hence, we obtain that $y(n+1)<y^{*}$. Inversely, the remaining part of this lemma is evident.

Lemma 4.3. If $\max _{1 \leq i \leq m} \tilde{x}_{i}(n+1)>\max _{1 \leq i \leq m} \tilde{x}_{i}(n)$, then $y(n+1)>y^{*}$. Inversely, if $y(n+1) \leq y^{*}$, then $\max _{1 \leq i \leq m} \tilde{x}_{i}(n+1) \leq$ $\max _{1 \leq i \leq m} \tilde{x}_{i}(n)$.

Proof. Assume that there exists a positive integer $1 \leq i_{0} \leq m$ such that $\tilde{x}_{i_{0}}(n+1)=\max _{1 \leq i \leq m} \tilde{x}_{i}(n+1)>\max _{1 \leq i \leq m} \tilde{x}_{i}(n)$. Then, $\tilde{x}_{i_{0}}(n+1)-\tilde{x}_{i_{0}}(n)>0$ and $\tilde{x}_{i_{0}}(n+1)-\tilde{x}_{i_{0}-1}(n)>0$, and by $(41)$, we have that

$$
0<\tilde{x}_{i_{0}}(n+1)-\tilde{x}_{i_{0}}(n) \leq \frac{a_{i_{0}}}{1-a_{i_{0}}}\left\{\frac{\tilde{\phi}_{i_{0}}(y(n+1))}{\tilde{\phi}_{i_{0}}\left(y^{*}\right)}-1\right\} \tilde{x}_{i_{0}}(n+1),
$$

from which we obtain that $\tilde{\phi}_{i_{0}}(y(n+1))>\tilde{\phi}_{i_{0}}\left(y^{*}\right)$. Hence, we obtain that $y(n+1)>y^{*}$. Inversely, the remaining part of this lemma is evident.

Lemma 4.4. If $R_{0}>1$, then any solution of system (41),

$$
\begin{aligned}
& \liminf _{n \rightarrow \infty} y(n) \geq h^{-1}\left(\alpha ; \bar{x}_{1}, \bar{x}_{2}, \ldots, \bar{x}_{m}\right)>0, \\
& \liminf _{n \rightarrow \infty} x_{i}(n) \geq \underline{b}^{m-1}(1-\bar{a})^{l_{0}} x_{i}^{*}>0, \quad 1 \leq i \leq m,
\end{aligned}
$$

where the integer $l_{0} \geq m-1$ is sufficiently large such that

$$
y^{*}<y^{\Delta}:=k\left\{1-(1-\beta)^{l_{0}}\right\}, \quad k=\frac{\alpha}{\beta}-\frac{1}{\beta} \sum_{i=1}^{m} \psi_{i}\left(y^{*}\right) r x_{i}^{*}>y^{*},
$$

and

$$
\bar{a}=\max _{1 \leq i \leq m} a_{i}, \quad \underline{b}=\min _{1 \leq i \leq m}\left(\frac{\tilde{\phi}_{i}(\underline{y})}{\tilde{\phi}_{i}\left(y^{*}\right)}, 1-a_{i}\right)<1 .
$$

Proof. For any fixed nonnegative constants $x_{1}, x_{2}, \ldots, x_{m}$,

$$
h\left(y ; x_{1}, x_{2}, \ldots, x_{n}\right) \equiv \beta y+\sum_{i=1}^{m} \psi_{i}(y) x_{i},
$$

is a strictly monotone increasing function of $y$, and for a positive constant $z$, there exists the inverse function $y=$ $h^{-1}\left(z ; x_{1}, x_{2}, \ldots, x_{m}\right)$ of $z=h\left(y ; x_{1}, x_{2}, \ldots, x_{n}\right)$. Then, by Lemmas 2.2 and 2.4 , we obtain that $\bar{x}_{1}, \bar{x}_{2}, \ldots, \bar{x}_{m}$ are uniformly bounded and

$$
\underline{y} \geq h^{-1}\left(\alpha ; \bar{x}_{1}, \bar{x}_{2}, \ldots, \bar{x}_{m}\right)>0 .
$$

We first prove the claim that any solution $\left(y(n), x_{i}(n), x_{2}(n), \ldots, x_{m}(n)\right)$ of system (10) does not have the following property: for any $0<r<1$, there exists a nonnegative integer $n_{0}$ such that $x_{i}(n) \leq r x_{i}^{*}, 1 \leq i \leq m$ for all $n \geq n_{0}$. Suppose on the contrary that there exist a solution $\left(y(n), x_{1}(n), x_{2}(n), \ldots, x_{m}(n)\right)$ of system (10) and a nonnegative integer 
$n_{0}$ such that $x_{i}(n) \leq r x_{i}^{*}, 1 \leq i \leq m$ for all $n \geq n_{0}$. One can represent the first equation of (10) in the form of the following Volterra difference equations (see for example [1,2]):

$$
y(n+1)=\frac{\alpha}{\beta}\left\{1-(1-\beta)^{n+1-n_{0}}\right\}+(1-\beta)^{n+1-n_{0}} y\left(n_{0}\right)-\sum_{l=n_{0}+1}^{n+1}(1-\beta)^{n+1-l} \sum_{i=1}^{m} \psi_{i}(y(l)) x_{i}(l-1) .
$$

Let $k=\frac{\alpha}{\beta}-\frac{1}{\beta} \sum_{i=1}^{m} \psi_{i}\left(y^{*}\right) r x_{i}^{*}$. Then, by $y^{*}=\frac{\alpha}{\beta}-\frac{1}{\beta} \sum_{i=1}^{m} \psi_{i}\left(y^{*}\right) x_{i}^{*}$ and $k>y^{*}$, there exists a sufficiently large integer $l_{0} \geq m-1$ such that $k\left\{1-(1-\beta)^{l_{0}}\right\}>y^{*}$. Suppose that $y(n) \leq y^{*}$ for any $n_{0}+1 \leq n \leq n_{0}+l_{0}$. Then, by (47), and $y\left(n_{0}\right)>0$, we have that

$$
\begin{aligned}
y\left(n_{0}+l_{0}\right) & =\frac{\alpha}{\beta}\left\{1-(1-\beta)^{l_{0}}\right\}+(1-\beta)^{l_{0}} y\left(n_{0}\right)-\sum_{l=n_{0}+1}^{n_{0}+l_{0}}(1-\beta)^{n_{0}+l_{0}-l} \sum_{i=1}^{m} \psi_{i}(y(l)) x_{i}(l-1), \\
& >\frac{\alpha}{\beta}\left\{1-(1-\beta)^{l_{0}}\right\}-\frac{1-(1-\beta)^{l_{0}}}{\beta} \sum_{i=1}^{m} \psi_{i}\left(y^{*}\right) r x_{i}^{*} \\
& =k\left\{1-(1-\beta)^{l_{0}}\right\}>y^{*},
\end{aligned}
$$

which is a contradiction. Thus, there exists an integer $n_{1}$ such that $n_{0}+1 \leq n_{1} \leq n_{0}+l_{0}$ and $y\left(n_{1}\right)>y^{*}$. If $y(n)>y^{*}$ for some $n \geq n_{0}+1$, then by the first equation of (10), we have that,

$$
y(n+1)+\sum_{i=1}^{m} \psi_{i}(y(n+1)) r x_{i}^{*}>\alpha+(1-\beta) y^{*}=y^{*}+\sum_{i=1}^{m} \psi_{i}\left(y^{*}\right) x_{i}^{*},
$$

which is equivalent to

$$
y(n+1) \geq y^{\Delta}:=h^{-1}\left(y^{*}+\sum_{i=1}^{m} \psi_{i}\left(y^{*}\right) x_{i}^{*} ; r x_{1}^{*}, r x_{2}^{*}, \ldots, r x_{m}^{*}\right)>y^{*},
$$

because $h\left(y(n+1) ; r x_{1}^{*}, r x_{2}^{*}, \ldots, r x_{m}^{*}\right)>y^{*}+\sum_{i=1}^{m} \psi_{i}\left(y^{*}\right) x_{i}^{*}=h\left(y^{\triangle} ; r x_{1}^{*}, r x_{2}^{*}, \ldots, r x_{m}^{*}\right)$ and $h\left(y ; x_{1}, x_{2}, \ldots, x_{m}\right)$ is a strictly monotone increasing function of $y$.

Therefore, we may choose $n_{1}=n_{0}+l_{0}$ and obtain that

$$
y(n) \geq y^{\Delta}>y^{*}, \quad \text { for any } n \geq n_{0}+l_{0} .
$$

Then, by the second part of Lemma 4.2, we have that

$$
\min _{1 \leq i \leq m} \tilde{x}_{i}(n+1) \geq \min _{1 \leq i \leq m} \tilde{x}_{i}(n), \quad \text { for any } n \geq n_{0}+l_{0}-1 .
$$

Thus, we obtain that there exists a positive constant $\hat{\tilde{x}}$ such that $x_{i}(n) \geq \hat{\tilde{x}} x_{i}^{*}, \quad 1 \leq i \leq m$ for any $n \geq n_{0}+l_{0}-1$. Moreover, consider the sequence $\{w(n)\}_{n=n_{0}+l_{0}-1}^{\infty}$ defined by

$$
w(n)=\sum_{i=1}^{m} x_{i}(n)
$$

By $R_{0}>1$ with assumptions (40) and $y^{\triangle}>y^{*}$, we have that $-a_{i}+\phi_{i}\left(y^{\triangle}\right)>0,1 \leq i \leq m$. Then, from (11), we obtain that

$$
\begin{aligned}
w(n+1)-w(n) & \geq \frac{1}{1-\left(\min _{1 \leq i \leq m} a_{i}\right)} \sum_{i=1}^{m}\left\{-a_{i}+\phi_{i}\left(y^{\triangle}\right)\right\} x_{i}(n) \\
& \geq \frac{1}{1-\left(\min _{1 \leq i \leq m} a_{i}\right)} \sum_{i=1}^{m}\left\{-a_{i}+\phi_{i}\left(y^{\triangle}\right)\right\} \hat{\tilde{x}} x_{i}^{*} \\
& >0 \text { for any } n \geq n_{0}+l_{0}-1,
\end{aligned}
$$

which implies that $\lim _{n \rightarrow \infty} w(n)=+\infty$. However, by (50) and Lemma 2.3, it holds that there are a positive constant $n_{4} \geq n_{0}+l_{0}-1$ and $\bar{w}$ such that $w(n) \leq \bar{w}$ for any $n \geq n_{4}$, which leads to a contradiction. Hence, the claim is proved.

By the claim, we are left to consider the two possibilities for $\overline{\tilde{x}}(n)=\max _{1 \leq i \leq m} \tilde{x}_{i}(n)$. First, $\overline{\tilde{x}}(n) \geq r$ for all $n$ sufficiently large. Second, we consider the case that $\overline{\tilde{x}}(n)$ oscillates about $r$ for all sufficiently large $n$. If the first condition that $\overline{\tilde{x}}(n) \geq r$ holds for all sufficiently large $n$, then we get the conclusion of the proof. For the second case that $\overline{\tilde{x}}(n)$ oscillates about $r$ for all sufficiently large $n$, let two sequences $\left\{\underline{n}_{k}\right\}_{k=1}^{\infty}$ and $\left\{\bar{n}_{k}\right\}_{k=1}^{\infty}$ be such that

$$
\overline{\tilde{x}}\left(\underline{n}_{k}-1\right), \overline{\tilde{x}}\left(\bar{n}_{k}+1\right)>r, \quad \text { and } \quad \overline{\tilde{x}}(n) \leq r \quad \text { for any } \underline{n}_{k} \leq n \leq \bar{n}_{k}, k=1,2, \ldots
$$


If $\bar{n}_{k}-\underline{n}_{k} \geq l_{0}$ for some $k \geq 1$, then by the above discussion from (47) to (48), replacing $n_{0}$ by $\underline{n}_{k}$, we obtain that $y(n) \geq y^{\Delta}>y^{*}$ for any $\underline{n}_{k}+l_{0} \leq n \leq \bar{n}_{k}$ and by Lemma 4.2 , we have that

$$
\min _{1 \leq i \leq m} \tilde{x}_{i}(n) \geq \min _{1 \leq i \leq m} \tilde{x}_{i}(n-1), \quad \text { for any } \underline{n}_{k}+l_{0} \leq n \leq \bar{n}_{k} .
$$

Therefore, we need to estimate the lower bound of $\min _{\underline{n}_{k} \leq n \leq \underline{n}_{k}+l_{0}-1}\left(\min _{1 \leq i \leq m} \tilde{x}_{i}(n)\right)$.

Put $\tilde{x}_{0}(n)=\tilde{x}_{L}(n), n=0,1, \ldots$ Then, by $(41)$, we have that

$$
\left\{\begin{array}{l}
\tilde{x}_{i}(n+1) \geq\left(1-a_{i}\right) \tilde{x}_{i}(n), \\
\tilde{x}_{i}(n+1) \geq \frac{\tilde{\phi}_{i}(\underline{y})}{\tilde{\phi}_{i}\left(y^{*}\right)} \tilde{x}_{i-1}(n), \quad i=1,2, \ldots, m, n=0,1,2, \ldots
\end{array}\right.
$$

Therefore, we have that

$$
\left\{\begin{array}{l}
\tilde{x}_{i}(n+1) \geq\left(1-a_{i}\right) \tilde{x}_{i}(n+1-l), \quad l=0,1,2, \ldots, n+1, i=1,2, \ldots, m, \\
\tilde{x}_{i}(n+1) \geq\left(\prod_{k=j+1}^{i} \frac{\tilde{\phi}_{k}(\underline{y})}{\tilde{\phi}_{k}\left(y^{*}\right)}\right) \tilde{x}_{j}(n+1-(i-j)), \quad \text { for any } m \geq i>j \geq 0 .
\end{array}\right.
$$

Then, for any $\bar{n}_{k-1}+1 \leq n \leq \underline{n}_{k}-1$, there exists a $1 \leq i_{0} \leq m$ such that $\tilde{x}_{i_{0}}(n) \geq r$ and, hence, we can easily obtain that for any $1 \leq i \leq m$,

$$
\tilde{x}_{i}(n+m-1) \geq \underline{b}^{m-1} r, \quad i=1,2, \ldots, m,
$$

and

$$
\tilde{x}_{i}(n+l) \geq \underline{b}^{m-1}(1-\bar{a})^{l-(m-1)} r, \quad l=m, m+1, \ldots, i=1,2, \ldots, m .
$$

In particular, for any $i=1,2, \ldots, m$, we have that

$$
\left\{\begin{array}{l}
\tilde{x}_{i}(n) \geq \underline{b}^{m-1} r, \quad \bar{n}_{k-1}+m \leq n \leq \underline{n}_{k}+m-2, \\
\text { and } \\
\tilde{x}_{i}(n) \geq \underline{b}^{m-1}(1-\bar{a})^{n-\left(\underline{n}_{k}+m-2\right)} r, \quad \underline{n}_{k}+m-1 \leq n .
\end{array}\right.
$$

Thus, by $l_{0} \geq m-1$,

$$
\tilde{x}_{i}(n) \geq \underline{b}^{m-1}(1-\bar{a})^{l_{0}-1-(m-2)} r, \quad \bar{n}_{k-1}+m \leq n \leq \bar{n}_{k}, \quad i=1,2, \ldots, m .
$$

Then,

$$
\tilde{x}_{i}(n) \geq \underline{b}^{m-1}(1-\bar{a})^{l_{0}} r, \quad \bar{n}_{k}+1 \leq n \leq \bar{n}_{k}+m-1, \quad i=1,2, \ldots, m .
$$

Hence, we obtain that

$$
\tilde{x}_{i}(n) \geq \underline{b}^{m-1}(1-\bar{a})^{l_{0}} r, \quad \bar{n}_{k-1}+m \leq n \leq \bar{n}_{k}+m-1, \quad i=1,2, \ldots, m .
$$

Since the above $k \geq 2$ is arbitrarily chosen, we conclude that for any $i=1,2, \ldots, m, x_{i}(n) \geq \underline{b}^{m-1}(1-\bar{a})^{l_{0}} r$ for all sufficiently large $n$ for the second case. Since $r(0<r<1)$ is also arbitrarily chosen, we conclude that

$$
\liminf _{n \rightarrow \infty} x_{i}(n) \geq \underline{b}^{m-1}(1-\bar{a})^{l_{0}} x_{i}^{*}, \quad 1 \leq i \leq m .
$$

This completes the proof.

\section{A special class of (10) where the endemic equilibrium is globally asymptotically stable for $R_{0}>1$}

Assume $R_{0}>1$. Then, by Lemma 4.4, the system (41) is permanent and by Theorem 3.1, system (41) has a unique endemic equilibrium $E^{*}=\left(y^{*}, x_{1}^{*}, x_{2}^{*}, \ldots, x_{m}^{*}\right)$. Thus, (41) has a unique endemic equilibrium $\tilde{E}^{*}=\left(y^{*}, \tilde{x}_{1}^{*}, \tilde{x}_{2}^{*}, \ldots, \tilde{x}_{m}^{*}\right)$ with $\tilde{x}_{1}^{*}=\tilde{x}_{2}^{*}=\cdots=\tilde{x}_{m}^{*}=\tilde{x}^{*}=1$.

In the rest of this paper, extending the result in Muroya et al. [13] to the following special class of (10), we show that the endemic equilibrium $\tilde{E}^{*}$ of (41) is globally asymptotically stable. Assume that

$$
b_{i}=b>0 \text { or } 0, \quad i=1,2, \ldots, m,
$$

and $b_{0}=b_{L}, x_{0}^{*}=x_{L}^{*}$ and there is a strictly monotone increasing function $\psi(y)$ on $y \geq 0$ and $\psi(0)=0$ such that for $i=1,2, \ldots, m$, 


$$
\left\{\begin{array}{l}
\psi_{i}(y)=b_{i} \psi(y), \\
\hat{\tilde{\psi}}_{i}(y)= \begin{cases}\frac{\psi(y)}{\psi\left(y^{*}\right)}, & \text { if } b_{i} \neq 0 \\
1, & \text { if } b_{i}=0\end{cases} \\
\phi_{i}(y)=\frac{a_{i} x_{i}^{*}}{x_{i-1}^{*}} \hat{\tilde{\psi}}_{i-1}(y)
\end{array}\right.
$$

and $\hat{\tilde{\psi}}_{0}(y)=\hat{\tilde{\psi}}_{L}(y), \tilde{x}_{0}(n)=\tilde{x}_{L}(n)$, and $\alpha>0,0<\beta<1$ and $0<a_{i}<1, i=1,2, \ldots, m$ are constants. Then, this system of (10) is equivalent to the following special class of difference equations:

$$
\left\{\begin{array}{l}
y(n+1)-y(n)=\frac{1}{1-\beta}\left\{\alpha-\beta y(n+1)-\psi(y(n+1)) \sum_{i=1}^{m} b_{i} \tilde{x}_{i}(n)\right\}, \\
\tilde{x}_{1}(n+1)-\tilde{x}_{1}(n)=\frac{a_{1}}{1-a_{1}}\left\{\hat{\tilde{\psi}}_{L}(y(n+1)) \tilde{x}_{L}(n)-\tilde{x}_{1}(n+1)\right\}, \quad 1 \leq L \leq m, \\
\tilde{x}_{i}(n+1)-\tilde{x}_{i}(n)=\frac{a_{i}}{1-a_{i}}\left\{\hat{\tilde{\psi}}_{i-1}(y(n+1)) \tilde{x}_{i-1}(n)-\tilde{x}_{i}(n+1)\right\}, \quad i=2,3, \ldots, m, \quad n \geq 0 .
\end{array}\right.
$$

For example, Muroya et al. [13] treated the case that $b_{i}=0, i=1,2, \ldots, m-1$ and $b_{m}=b>0$.

Let the Lyapunov function (see $[11,12]$ ) be

$$
U(n)=\frac{1-\beta}{b \psi\left(y^{*}\right)} U_{y}(n)+\sum_{i=1}^{m} \frac{1-a_{i}}{a_{i}} U_{i}(n)+U_{+}(n),
$$

where

$$
\left\{\begin{array}{l}
U_{y}(n)=\int_{y^{*}}^{y(n)}\left(1-\frac{\psi\left(y^{*}\right)}{\psi(y)}\right) \mathrm{d} y, \quad U_{i}(p)=g\left(\tilde{x}_{i}(n)\right), \quad i=1,2, \ldots, m, \\
U_{+}(p)=\sum_{i=1}^{m} g\left(\tilde{x}_{i}(n)\right)
\end{array}\right.
$$

and $g(x)=x-1-\ln x, x>0$.

We now show that $U(n+1)-U(n) \leq 0$. First, we calculate $U_{y}(n+1)-U_{y}(n)$ by using the first equation of $(41)$ :

$$
\begin{aligned}
U_{y}(n+1)-U_{y}(n) & =(y(n+1)-y(n))-\int_{y(n)}^{y(n+1)} \frac{\psi\left(y^{*}\right)}{\psi(y)} \mathrm{d} y \\
& \leq(y(n+1)-y(n))-\psi\left(y^{*}\right) \frac{y(n+1)-y(n)}{\psi(y(n+1))} \\
& =\frac{\psi(y(n+1))-\psi\left(y^{*}\right)}{\psi(y(n+1))}(y(n+1)-y(n)) \\
& =\frac{\psi(y(n+1))-\psi\left(y^{*}\right)}{(1-\beta) \psi(y(n+1))}\left\{\alpha-\beta y(n+1)-\psi(y(n+1)) \sum_{i=1}^{m} b_{i} \tilde{x}_{i}(n)\right\},
\end{aligned}
$$

because by the fact that $\psi(y)$ is strictly monotone increasing on $y>0$, it holds that

$$
\int_{y(n)}^{y(n+1)} \frac{1}{\psi(s)} \mathrm{d} s \geq \frac{y(n+1)-y(n)}{\psi(y(n+1))} \text { for any } y(n)>0 \text { and } y(n+1)>0 .
$$

Substituting $\alpha=\beta y^{*}+\psi\left(y^{*}\right) \sum_{i=1}^{m} b_{i} \tilde{x}_{i}^{*}$ into (55) and by the definition $b_{i}, i=1,2, \ldots, m$, we have $b_{i}\left(1-\frac{\psi\left(y^{*}\right)}{\psi(y)}\right)=$ $b\left(1-\frac{1}{\hat{\tilde{\psi}}_{i}(y)}\right), i=1,2, \ldots, m$, and we obtain that

$$
\begin{aligned}
U_{s}(p+1)-U_{s}(p) \leq & \frac{\psi(y(n+1))-\psi\left(y^{*}\right)}{(1-\beta) \psi(y(n+1))} \\
& \times\left\{\beta\left(y^{*}-y(n+1)\right)+\psi\left(y^{*}\right) \sum_{i=1}^{m} b_{i}\left(\tilde{x}_{i}^{*}-\frac{\psi(y(n+1))}{\psi\left(y^{*}\right)} \tilde{x}_{i}(n)\right)\right\} \\
= & -\frac{\beta}{1-\beta} \frac{\left(\psi(y(n+1))-\psi\left(y^{*}\right)\right)\left(y(n+1)-y^{*}\right)}{\psi(y(n+1))} \\
& +\frac{\psi\left(y^{*}\right)}{1-\beta} \sum_{i=1}^{m} b\left(1-\frac{1}{\hat{\tilde{\psi}}_{i}(y(n+1))}\right)\left(1-\hat{\tilde{\psi}}_{i}(y(n+1)) \tilde{x}_{i}(n)\right) .
\end{aligned}
$$


Second, similarly, we calculate $U_{i}(n+1)-U_{i}(n), i=1,2, \ldots, m$ by using the second and third equations of (41):

$$
\begin{aligned}
U_{i}(n+1)-U_{i}(n) & =\left\{\tilde{x}_{i}(n+1)-\tilde{x}_{i}(n)\right\}-\ln \frac{\tilde{x}_{i}(n+1)}{\tilde{x}_{i}(n)} \\
& \leq\left\{\tilde{x}_{i}(n+1)-\tilde{x}_{i}(n)\right\}-\frac{\tilde{x}_{i}(n+1)-\tilde{x}_{i}(n)}{\tilde{x}_{i}(n+1)} \\
& =\frac{\tilde{x}_{i}(n+1)-1}{\tilde{x}_{i}(n+1)}\left(\tilde{x}_{i}(n+1)-\tilde{x}_{i}(n)\right) \\
& =\left\{\begin{array}{l}
\frac{a_{1}}{1-a_{1}}\left(1-\frac{1}{a_{i}}\right)\left\{\hat{\tilde{\psi}}_{L}(y+1)\right. \\
\frac{a_{i}}{1-a_{i}}\left(1-\frac{1}{\tilde{x}_{i}(n+1)}\right)\left\{\hat{\tilde{\psi}}_{i-1}(y(n+1)) \tilde{x}_{L-1}(n)-\tilde{x}_{1}(n+1)\right\}, \quad \text { if } i=1,
\end{array}\right.
\end{aligned}
$$

Finally, calculating $U_{+}(n+1)-U_{+}(n)$, we get that

$$
U_{+}(n+1)-U_{+}(n)=\sum_{i=1}^{m}\left\{g\left(\tilde{x}_{i}(n+1)\right)-g\left(\tilde{x}_{i}(n)\right)\right\} .
$$

Therefore, by the above discussion and (54), similar to McCluskey [11, Proof of Theorem 4.1], we obtain that

$$
\begin{aligned}
& U(n+1)-U(n) \leq-\frac{\beta}{\psi\left(y^{*}\right) \psi(y(n+1))}\left\{\psi(y(n+1))-\psi\left(y^{*}\right)\right\}\left(y(n+1)-y^{*}\right) \\
& +\sum_{i=1}^{m}\left(1-\frac{1}{\tilde{\tilde{\psi}}_{i}(y(n+1))}\right)\left(1-\hat{\tilde{\psi}}_{i}(y(n+1)) \tilde{x}_{i}(n)\right) \\
& +\sum_{i=1}^{m}\left(1-\frac{1}{\tilde{x}_{i}(n+1)}\right)\left\{\hat{\tilde{\psi}}_{i-1}(y(n+1)) \tilde{x}_{i-1}(n)-\tilde{x}_{i}(n+1)\right\} \\
& +\sum_{i=1}^{m}\left\{g\left(\tilde{x}_{i}(n+1)\right)-g\left(\tilde{x}_{i}(n)\right)\right\} \\
& =-\frac{\beta}{\psi\left(y^{*}\right) \psi(y(n+1))}\left\{\psi(y(n+1))-\psi\left(y^{*}\right)\right\}\left(y(n+1)-y^{*}\right) \\
& +\left\{\sum_{i=1}^{m}\left(1-\frac{1}{\hat{\tilde{\psi}}_{i}(y(n+1))}\right)-\sum_{i=1}^{m}\left(\hat{\tilde{\psi}}_{i}(y(n+1))-1\right) \tilde{x}_{i}(n)\right\} \\
& +\left\{\sum_{i=0}^{m-1}\left(\hat{\tilde{\psi}}_{i}(y(n+1))-1\right) \tilde{x}_{i}(n)+\sum_{i=0}^{m-1}\left(\tilde{x}_{i}(n)-\tilde{x}_{i}(n+1)\right)\right. \\
& \left.-\left(\sum_{i=0}^{m-1} \frac{\hat{\tilde{\psi}}_{i}(y(n+1)) \tilde{x}_{i}(n)}{\tilde{x}_{i+1}(n+1)}\right)+m\right\}+\sum_{i=1}^{m}\left\{g\left(\tilde{x}_{i}(n+1)\right)-g\left(\tilde{x}_{i}(n)\right)\right\} \\
& =-\frac{\beta}{\psi\left(y^{*}\right) \psi(y(n+1))}\left\{\psi(y(n+1))-\psi\left(y^{*}\right)\right\}\left(y(n+1)-y^{*}\right) \\
& -\left[\sum_{i=1}^{m} g\left(\frac{1}{\hat{\tilde{\psi}}_{i}(y(n+1))}\right)+\sum_{i=0}^{m-1}\left\{g\left(\tilde{x}_{i}(n)\right)-g\left(\tilde{x}_{i}(n+1)\right)\right\}\right. \\
& \left.+\sum_{i=0}^{m-1} g\left(\frac{\hat{\tilde{\psi}}_{i}(y(n+1)) \tilde{x}_{i}(n)}{\tilde{x}_{i+1}(n+1)}\right)\right]+\sum_{i=1}^{m}\left\{g\left(\tilde{x}_{i}(n+1)\right)-g\left(\tilde{x}_{i}(n)\right)\right\} \\
& =-\frac{\beta}{\psi\left(y^{*}\right) \psi(y(n+1))}\left\{\psi(y(n+1))-\psi\left(y^{*}\right)\right\}\left(y(n+1)-y^{*}\right) \\
& -\left[\sum_{i=1}^{m} g\left(\frac{1}{\hat{\tilde{\psi}}_{i}(y(n+1))}\right)+\sum_{i=0}^{m-1} g\left(\frac{\hat{\tilde{\psi}}_{i}(y(n+1)) \tilde{x}_{i}(n)}{\tilde{x}_{i+1}(n+1)}\right)\right] .
\end{aligned}
$$

Moreover, we have that

$$
\left(\psi(y(n+1))-\psi\left(y^{*}\right)\right)\left(y(n+1)-y^{*}\right) \leq 0,
$$


with equality, if and only if $y(n+1)=y^{*}$. Hence, $U(n+1)-U(n) \leq 0$ for any $n \geq 0$. Since $U(n) \geq 0$ is a monotone decreasing sequence, there is a $\operatorname{limit}_{n \rightarrow+\infty} U(n) \geq 0$. Then, $\lim _{n \rightarrow+\infty}(U(n+1)-U(n))=0$, from which we obtain that

$$
\lim _{n \rightarrow+\infty} y(n+1)=y^{*}, \quad \lim _{n \rightarrow+\infty} \tilde{x}_{i}(n+1)=\tilde{x}^{*}, \quad i=1,2, \ldots, m,
$$

that is, $\lim _{n \rightarrow+\infty}\left(y(n), x_{1}(n), x_{2}(n), \ldots, x_{m}(n)\right)=\left(y^{*}, x_{1}^{*}, x_{2}^{*}, \ldots, x_{m}^{*},\right)$. Since $U(n) \leq U(0)$ for all $n \geq 0$ and $g(x) \geq 0$ with equality if and only if $x=1, E^{*}$ is uniformly stable. Hence, the proof is complete.

\section{Conclusions}

In this paper, motivated by the recent progress of several proof techniques on the permanence and Lyapunov techniques (see for example, $[3,11,13,20]$ ), under the assumption that each $\psi_{i}(y)$ is a monotone increasing function of $y, 1 \leq i \leq m$, we prove the permanence of the system (9) treated in [9], and give a partial answer to the remaining open question for the case $R_{0}>1$ about the global asymptotic stability for the endemic equilibrium of the system (9).

In spite of the proofs of the main results in [21] making use of the theory of nonnegative matrices, Lyapunov functions and a subtle grouping technique in estimating the derivatives of Lyapunov functions guided by graph theory, we apply the techniques of Lyapunov functions in [11] and [13] to prove the global asymptotic stability for the endemic equilibrium of system (7) for the case $R_{0}>1$ which is simple and we no longer need to use any of the theory of nonnegative matrices and graph theory (cf. [6]). Moreover, we offer new techniques to obtain lower bounds for the permanence of group epidemic models which will be useful in applications (cf. persistence theory in dynamical systems, for example, [4,6,17]). Our future work will be to apply these techniques to other types of not only discrete but also continuous group epidemic models.

\section{Acknowledgement}

The first author's research is partially supported by Scientific Research (c), No. 21540230 of the Japan Society for the Promotion of Science.

\section{References}

[1] M.R. Crisci, V.B. Kolmanovskii, E. Russo, A. Vecchio, Boundedness of discrete Volterra equations, J. Math. Anal. Appl. 211 (1997) 106-130.

[2] S.N. Elaydi, An Introduction to Difference Equations, Springer-Verlag, 1996.

[3] Y. Enatsu, Y. Nakata, Y. Muroya, Global stability for a class of discrete SIR epidemic models, Math. Biosci. Eng. 7 (2010) 347-361.

[4] H.I. Freedman, M.X. Tang, S.G. Ruan, Uniform persistence of flows near a closed positively invariant set, J. Dynam. Differential Equations 6 (1994) $583-600$.

[5] H. Guo, M.Y. Li, Global dynamics of a stage progression model for infectious disease, Math. Biosci. Eng. 3 (2006) 513-525

[6] H. Guo, M.Y. Li, Z. Shuai, Global stability of the endemic equilibrium of multigroup SIR epidemic models, Can. Appl. Math. Q. 14 (2006) $259-284$.

7] H.W. Hethcote, The mathematics of infections diseases, SIAM Rev. 42 (2000) 599-653.

[8] G. Izzo, Y. Muroya, A. Vecchio, A general discrete time model of population dynamics in the presence of an infection, Discrete Dyn. Nat. Soc. (2009), Article ID 143019, 15 pages. doi:10.1155/2009/143019.

[9] G. Izzo, A. Vecchio, A discrete time version for models of population dynamics in the presence of an infection, J. Comput. Appl. Math. 210 (2007) $210-221$.

[10] S. Jang, S.N. Elaydi, Difference equations from discretization of a continuous epidemic model with immigration of infectives, MTBI Cornell University Technical Report, 92 (2004)

[11] C.C. McCluskey, Complete global stability for an SIR epidemic model with delay-Distributed or discrete, Nonlinear Anal. RWA. 11 (2010) 55-59.

[12] C.C. McCluskey, Global stability for an SIR epidemic model with delay and nonlinear incidence, Nonlinear Anal. RWA 11 (2010) $3106-3109$.

[13] Y. Muroya, Y. Enatsu, G. Izzo, A. Vecchio, Global stability for a discrete SIR epidemic model with latency spreading in a heterogeneous host population (submitted for publication).

[14] A. Ramani, A.S. Carstea, R. Willox, B. Grammaticos, Oscillating epidemic: a discrete-time model, Physica A 333 (2004) $278-292$.

[15] M. Sekiguchi, Permanence of some discrete epidemic models, Int. J. Biomath. 2 (2009) 443-461.

[16] M. Sekiguchi, E. Ishiwata, Global dynamics of a discretized SIRS epidemic model with time delay, J. Math. Anal. Appl. 371 (2010) 195-202.

[17] H.R. Thieme, Mathematics in Population Biology, Princeton University Press, Princeton, 2003.

[18] P. van den Driessche, L. Wang, X. Zou, Modeling disease with latency and relapse, Math. Biosci. Eng. 4 (2007) $205-219$.

[19] L. Wang, M.Y. Li, D. Kirschner, Mathematical analysis of the global dynamics of a model for HTLV-1 infection and ATL progression, Math. Biosci. 179 (2002) 207-217.

[20] W. Wang, Global behavior of an SEIRS epidemic model with time delays, Appl. Math. Lett. 15 (2002) 423-428.

[21] Z. Yuan, X. Zou, Global threshold property in an epidemic model for disease with latency spreading in a heterogeneous host population, Nonlinear Anal. RWA 11 (2010) 3479-3490. 